\title{
DESIGNING A TEMPORARY COLD PROTECTIVE GEAR CONSTRUCTED BY FOLDING NEWSPRINT PAPER CONSIDERING HEAT-RETAINING PROPERTY AND RIGIDITY
}

\author{
Oda, Tatsuya (1); \\ Wesugi, Shigeru (2) \\ 1: Graduate School of Creative Science and Engineering, Waseda University; \\ 2: Faculty of Science and Engineering, Waseda University
}

\begin{abstract}
During the cold season, the cold protective products are often short during evacuation life after a natural disaster. If evacuees can make and wear simple cold protective gears by using materials obtainable on site, it will reduce the burden on the evacuees in emergent situation. Therefore, we investigated the structure constructed by folding newsprint paper, which can improve the heat retention effect and be applied to various body shapes. Focusing on the glide reflection structure repeating a smaller chamber, the basic size was determined by experiments with reference to the accordion shape, and the experimental results indicated that the heat retention effect was significantly greater than that of a mere air layer and those of ordinary fabrics. Next, it was found that the apex angle of structure had no significant difference in the heat retention effect. Then, the dimensions of the structure were determined to maintain the air layer under the pressure of the clothes by simulation of structural analyses. Finally, we made a temporary cold protective gear that can practically cover the trunk of the body and found that the heat retention effect was significantly higher than that of unprocessed newsprint and that of accordion shape.
\end{abstract}

Keywords: Experience design, Open source design, Research methodologies and methods, Temporary cold protective gear, Newsprint paper

\section{Contact:}

Wesugi, Shigeru

Waseda University

Faculty of Science and Engineering

Japan

wesugi@waseda.jp 


\section{INTRODUCTION}

In recent years, there have been a lot of design-based approaches to solving problems related to performing daily activities of life, such as securing drinking water and generating electricity, in developing regions where water and electricity infrastructures are inadequate (Smith, 2007). The approach of solving problems with locally available materials and fabrication can be effective in times of large-scale natural disaster even in industrialised countries with well-developed infrastructures.

Japan often suffers from large natural disasters, including river flooding and landslide damage caused by heavy rainfall. As results, many evacuees are forced to live in evacuation centres. About $30 \%$ of disasterrelated deaths resulted from physical and mental fatigue due to living in evacuation centres. The poor living condition in evacuation centres is a serious problem (Reconstruction Agency, 2012). One of the reasons is considered to be short of necessary supplies during the evacuation life. The authors focus on the survey results of short commodity related to evacuation life and support among those who were staying in evacuation centres two weeks after the Great East Japan Earthquake (quantity of responses = 284) (Cabinet Office, 2013-2). We organized each item as common item and the high common items are: fuel such as gasoline and kerosene (261), cold protective products such as blankets, mats and tatami mats (207), underwear and clothes (164), drinking water (126), and batteries and chargers (116).

These listed items are those that are routinely available through the social infrastructures such as water, electricity and logistics. An evacuation life is a situation in which the existing infrastructures are no longer functioning, practically a situation similar to a developing area where insufficient infrastructures exist. Therefore, ideas of livelihood support design for developing areas can also be used sufficiently for evacuation life. In the design-based approaches, there are tools and devices that are available at very low cost, such as the " $\$ 100$ laptop", and those that can be manufactured and maintained locally, such as the thermally efficient wood stove. In the case of evacuation life, the latter tools and devices are effective that can be fabricated on site, using locally available materials. In addition, it's enough to act as a bridge until supplies arrive or infrastructure is re-established.

In this paper, we focus on cold protective products, which are high on the list of short commodities. Some of the cold protective products can be bulky at the time of storage, and it may not be possible to stockpile more than the expected number of evacuees, which may cause a shortage of supplies depending on the evacuation situations. During the first three days of a disaster, life-saving and rescue operations take precedence over supplying relief materials (Committee of Infrastructure Planning and Management, 2016), and evacuees need to rely on stockpiles. Therefore, it is necessary to prepare for possible disasters on their own including a whole week's stockpile and to protect themselves during a disaster, rather than relying completely on the national and local governments (Cabinet Office, 2013-1). Generally, elementary and junior high schools, gymnasiums and community centres are used as evacuation shelters. These facilities are not built for residential purpose and have low thermal insulation performance, additionally, they tend to be cold and hard to keep warm due to large space. The above indicates that the capacity of the stockpile is limited, and it is difficult to meet all individual requirements for keeping off the cold, especially during the cold season and in some cold regions.

If a body temperature decreases due to cold weather, negative effects including lowered immunity, poor blood circulation and autonomic nerve imbalance can occur. In addition, it has been reported that an increase of $1{ }^{\circ} \mathrm{C}$ in air temperature below $20^{\circ} \mathrm{C}$ reduces heat production to regulate body temperature by $4-5 \%$ (Edholm, 1978). Therefore, even a $1{ }^{\circ} \mathrm{C}$ increase in air temperature is significant.

In order to produce variety of protective measures against the cold in evacuation centres, this study designs the basic structure of a temporary cold protective gear constructed by folding newsprint paper which can be obtainable during a disaster and discusses scenarios that the idea can be used on site.

\section{METHOD}

\subsection{Related works}

Corrugated fiberboard is one of the materials that are relatively easy to obtain and shape even in evacuation centres. Corrugated fiberboard has superior characteristics, such as thermal insulation effect, high rigidity, low cost, ease of mass production, and often has been used as partitions and beds in shelters. The disaster prevention agreement allows the corrugated board manufacturing industry to provide standardised boards for bed. The agreement also indicates, even if a corrugated board factory is damaged, undamaged other factories around the disaster area can supply the boards. 
The authors focused on newsprint paper as an alternative material from the perspective of easily working on. The current number of newsprint paper is declining these days, however, it can be considered to be a material, in a sense, dispersed and stored by households because there is a system collecting regularly used paper. Moreover, it is easy to change shape and cut by hand without using tools.

The Tokyo Metropolitan Government published the disaster prevention book, that recommends the use of newsprint paper as wisdom for living during a disaster, such as putting one's feet in the bag stuffed with balled-up papers, or by wrapping paper around the body trunk for keeping off the cold (Tokyo Metropolitan Government, 2015). Although the empirical heat retention effect by using newsprint paper is well known, the quantitative effects and more efficient usages are not obvious yet. Therefore, the authors intended to study the structure to increase the thermal effect from the view of engineering design with as few sheets as possible and compare it with empirical use (Hodozuka, 2016, 2018).

The heat retention effect in use of newsprint paper is based on the property of air, which is difficult to transfer heat to. The thermal conductivity of still air, which indicates the ability of heat transfer within material, is relatively lower than that of other materials. Feathers and fabrics for clothing and glasswool insulation for building materials utilize this air property of resistance to heat transfer. In fabrics, each fibre makes many fine diamond shapes, which hold air in them. When wearing clothing, the air warmed by the body heat stays inside the fabric. The small volume of air trapped inside the diamond shape prevents convection and heat release from the human body. Moreover, in fabrics, a further volume of air is added when wearing clothes in layers. The building heat insulation material traps air in fine grains, which prevent convection and provide high heat retention. Newsprint paper can also be recycled as a heat insulator, such as cellulose fibre, which is shredded and chemically treated. However, a process such as shredding is not practical in evacuation centres. In particular, considering the limited availability of tools in evacuation centres, it is necessary to be able to create the specific structures of newsprint paper with less effort.

Therefore, we focused on an accordion shape with alternating upward and downward folds to manually make a partition that prevents air movement without complicated cutting or wasting paper chips. In addition, it can be recycled as newsprint, as it only needs to be folded without glues. The effects on heat retention were then compared among the accordion shape, the layered, the perforated, and balled-up wrinkled conditions of multiple sheets of newsprint paper. There was no significant difference in temperature when the number of sheets was layered 1-6, meanwhile, it indicated that the temperature difference, i.e., the heat retention effect, was increased by the accordion shape and wrinkled condition (Hodozuka, 2016). The practical application of these structures is to put them between clothes. If a mere wrinkled or accordion shape is placed between clothes, the structure will be crushed by the pressure of the clothes. Therefore, as shown in Figure 1, we focused on a folded-plate structure in which three accordion surfaces are combined at a fixed angle. This folded-plate structure is based on the principle of increasing the strength of paper by creasing it, and has also been applied to buildings. The dimensions of the structure were calculated by computer simulation to ensure that it has sufficient rigidity to withstand the pressure of the clothes, and the actual structure was made to investigate its heat retention effect. The results show that the paper structure retains heat better than the same number of unprocessed newsprint paper. Furthermore, when these two folded-plate ends were combined to wrap around the body like a belly band, the structure can be held in place during daily movements (Hodozuka, 2018).

\subsection{Idea}

This study aims to investigate the basic structure of shaped newsprint paper as a temporary cold protective gear. The basic structure is based on the accordion shape, which is not complicated and does not waste paper during processing. The basic structure is required to have a high rigidity that holds the air layer to be maintained during use even with a small number of sheets, and the structure is also required to be flexible enough to fit various body shapes and to follow the movements of the body.

Since the folded-plate structure is U-shaped with straight lines, it is easy to make gaps around the body when covering the trunk of the body. Therefore, it is necessary to divide it into small sections to adapt to various body shapes. In order to improve the heat retention effect as a temporary cold protective gear and make it adaptable to more people, we focused on "tessellation" in origami.

Origami, which is a traditional Japanese art of paper folding, has been advanced to origami engineering by including mathematical modelling and engineering applications. By creasing thin sheets, bending and deformation during folding can be restricted, and the direction of expansion and contraction can be changed according to the fold line. This makes it possible to create structures that are folded small and 
expanded large, and to limit the movement of the structure after expansion. For example, the idea of structures that can expand and contract have been researched and applied to solar panels for satellites and stent grafts for expanding blood vessels (Turner, 2016).

Since the tessellation is a method of repeatedly shaping small chambers of paper in a regular pattern, we thought that it would be possible to increase the rigidity of the paper structure while maintaining the air layer. In addition, in order to adapt the structures to variety of body shapes and body sizes, we designed a relatively small unit and combined them. Then, we focused on the glide reflection as shown in Figure 2, which is a structure that repeats the basic fold pattern in translation and inversion. Especially we chose the basic pattern for making origami balloon. This glide reflection structure is expected to increase rigidity while retaining an air layer separated by multiple folds. In addition, there are folds in the y-axis direction in Figure 2, which allow for bending around the y-axis. This indicates the structure deforms and covers along the surface of the body. By overlapping the ends of the structure on each other, the length of structure can be adjusted to any length, like a belt. This structure is shaped by repeating a single fold pattern without complicated cutting or wasting paper chips, therefore, the structure can be made in an evacuation shelter.

In this study, we design the glide reflection structure as the above shows. When used, this structure is placed between clothes, and the ends of the structure are connected to each other to cover the trunk of body, or to wrap arms and legs.

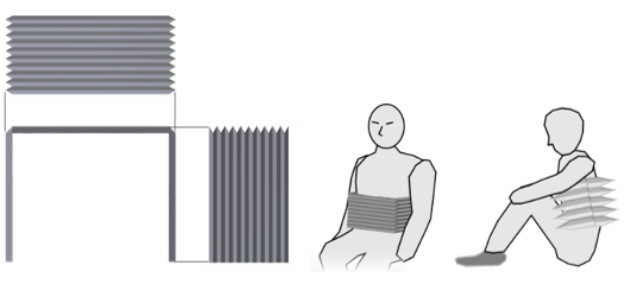

Figure 1. A folded-plate structure for temporary cold protective gear using newsprint paper

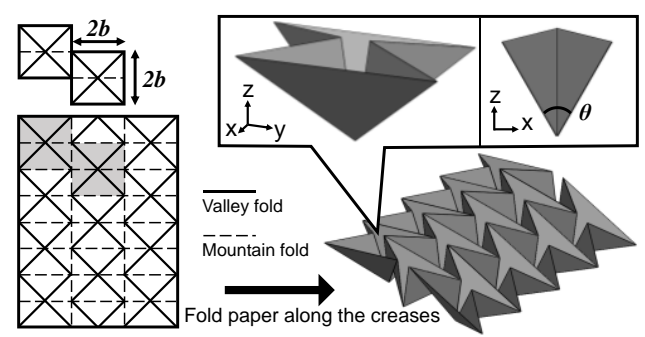

Figure 2. A glide reflection structure and its geometric net

\section{DESIGN}

The basic structure was determined by the following procedure with reference to the previous experimental one (Hodozuka, 2016, 2018), and a temporary cold protective gear was designed. The glide reflection structure proposed in 2.2 is designed by three conditions: the length of the fold line width $b$, the angle $\theta$ of the folded face to face as shown in Figure 2, and the size constraint of the newsprint paper (approx. $820 \times 540 \mathrm{~mm}$ for one spread). Especially, the length of the fold width $b$ is important because it determines the main shape of the structure.

First, the basic dimensions of the structure are studied from the perspective of heat retention. Next, on the basis of its dimensions, the rigidity of the structure, which is mainly to be maintained by the pressure of the clothes, is considered.

\subsection{Design of basic dimensions focused on heat retention}

\subsubsection{Design variables}

First of all, the dimensions of the structure are narrowed down from the point of view of heat retention, which is the most important performance of a temporary cold protective gear. As explained in 2.1, an air layer is necessary to improve the heat retention effect. However, it is reported that, when the air layer is thicker than $2 \mathrm{~cm}$, convection occurs and the insulation performance is not improved (Saito, 1974).

In the present study, the air layer is divided by folds in order to increase the rigidity and to improve the heat retention effect by preventing convection from occurring. Therefore, we experimentally investigated the relationship between the size of the chamber and the heat retention effect.

In order to determine the basic size of the chamber, the test pieces are an accordion shape of newsprint paper, which is a simple structure to separate the air layers and has been studied in previous experiments. We tested the heat retention effect depending on the size of the air layer by varying the width of the accordion shape. On the basis of the results, we next tested the heat retention effect with a specimen of a glide reflection structure of the same width as the accordion shape. Finally, we investigated the heat 
retention effect depending on the angle of the folded face to face of the accordion shape and the glide reflection structure.

\subsubsection{Experimental method}

The experimental method was based on the heat retaining test in the testing methods for woven and knitted fabrics by JIS and the authors' previous experiments (Hodozuka, 2016, 2018) on heat retention effect. In the method, a test piece covers a heat generator and the air temperature rise is measured due to heat transferred from the heat generator through the test piece. Therefore, the higher the heat retention of the test piece, the lower the temperature rise due to the transferred heat.

In this experiment, we investigated the temperature rise, the difference between thermocouple (1) and (2), $T_{0}={ }^{\circ} \mathrm{C}$ measured without the test piece and the temperature rise $T^{\circ} \mathrm{C}$ measured with the test piece, and compared the temperature rise difference $T-T_{0}={ }^{\circ} \mathrm{C}$ of the heat prevented from moving by the test pieces. Figure 3 shows the experimental environment, a hot plate (AS ONE Corporation, resolution $0.1{ }^{\circ} \mathrm{C}$ ) as a heat generator was located in a thermostatic bath (Yamato Scientific Co., Ltd. IN802, temperature control: $\pm 1.0^{\circ} \mathrm{C}$ ) in which the temperature was measured with a thermocouple (1) (T-type, range -50 to $+250{ }^{\circ} \mathrm{C}$, accuracy $\pm 0.5^{\circ} \mathrm{C}$ class 1 , resolution $0.1{ }^{\circ} \mathrm{C}$ ). The top of the hot plate is covered with the test piece and with an acrylic cover $(200 \times 200 \times 130 \mathrm{~mm})$ attaching a thermocouple (2) inside. The temperatures in the thermostatic bath and the hot plate were set at 15 and $36^{\circ} \mathrm{C}$ respectively, to simulate the environment of the shelter during the cold season. The test pieces were shaped of newsprint paper and were put into between cotton and knitwear, simulated to underwear and outerwear respectively. In addition, the acrylic cover prevents the cold air outside from mixing with the warm air inside. The thermostatic bath operates in a cycle of running, pausing, running, and pausing to reduce frost. The temperature was measured in $2 \mathrm{~h}$ after the switch from running to pausing in order to be measured on the same condition.

\subsubsection{Experiment and results on the basic size of an accordion shape}

In order to determine the size of the basic structure, test pieces of accordion shaped newsprint paper were compared with widths $a$ of 10,30, 50, and $70 \mathrm{~mm}$ (Figure 4 up). To prevent the accordion shaped structure from being crushed by the self weight as the width of the structure was increased, acrylic ribs support the paper structure without separating the air layer in all three conditions except the width 10 $\mathrm{mm}$ (Figure 4 down). Three test pieces were tested for each condition. Figure 5 shows the results of the experiment. The error bar indicates standard deviation. A comparison of the newsprint paper-free, newsprint paper-unprocessed, and accordion shaped newsprint paper showed a significant difference in temperature among the accordion shaped conditions and others. It was shown that the accordion shaped structure enhances the heat retention effect. Although there was no significant difference among the conditions with different accordion shape widths, there was a tendency for the temperature difference to be larger at 30 and $50 \mathrm{~mm}$ than in the other conditions.

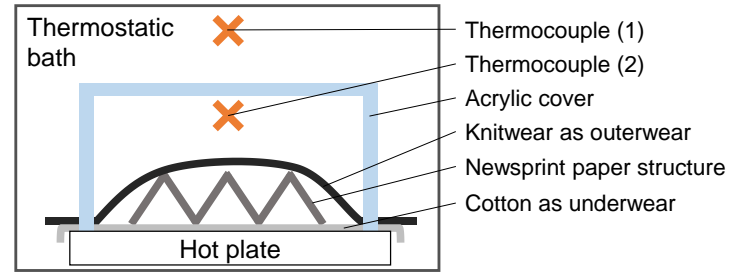

Figure 3. Experimental environment for measuring heat retention effect

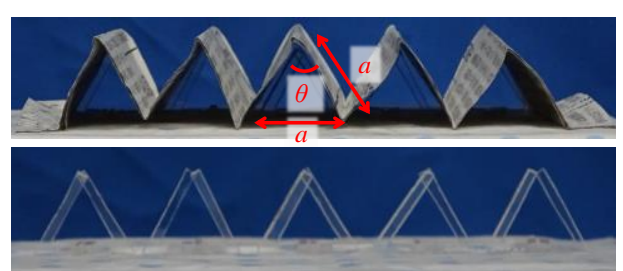

Figure 4. A test piece of accordion shaped newsprint paper and acrylic ribs

\subsubsection{Experiment and results on the glide reflection structure}

Next, we investigated the heat retention effect of newsprint paper shaped with glide reflection based on the method explained in 3.1.2. The test pieces were (i) glide reflection newsprint paper of $30 \mathrm{~mm}$ width, the same length as (ii), (ii) accordion shaped newsprint paper of $30 \mathrm{~mm}$ width, relatively high heat retention effect in 3.1.3, (iii) a hollow layer with a height of $26 \mathrm{~mm}$, the same height of (ii), in the condition, small wooden prisms $(10 \times 10 \times 26 \mathrm{~mm})$ are placed at the four corners and centre of the hot plate and support the cloth (knit) over the plate, (iv) $100 \%$ cotton knit with brushed backside, (v) survival sheet. Only under the condition (ii), the ribs were used to prevent the structure from collapsing. Under conditions (iv) and (v), the hot plate was covered with a cloth (cotton), cloth (knit), and test piece, in that order, in order to simulate the actual use of wearing a cotton knit or survival sheet over a jacket. 
Three test pieces were tested for each condition. Figure 6 shows the experimental results and the error bar indicates standard deviation. The results indicate that significant temperature differences occur in (iv) knit rather than (v) survival sheet, in (iii) hollow rather than (v) survival sheet, and in (i) glide reflection structure and (ii) accordion shape rather than the others. However, there was no significant difference among (i) glide reflection structure and (ii) accordion shape. In (iii) hollow layer condition, the more air layers than in survival sheets, the less heat-transmitting effect is likely to have worked. In addition, as it is reported that convection occurs when the air layer becomes thicker than $2 \mathrm{~cm}$ and the thermal insulation performance does not improve (Saito, 1974), it is thought that the air movement is suppressed in the paper structure by separating the air layer and thus the heat retention effect is functional.

The results suggest that a glide reflection structure has the same level of heat retention as that of accordion shape. The $30 \mathrm{~mm}$ wide accordion shaped structure cannot stand without ribs, which is not appropriate considering the practical application. For this reason, no ribs are required and the following experiments were conducted with width $a=15 \mathrm{~mm}$, which can maintain the structure under the pressure of the clothes in the previous experiments.

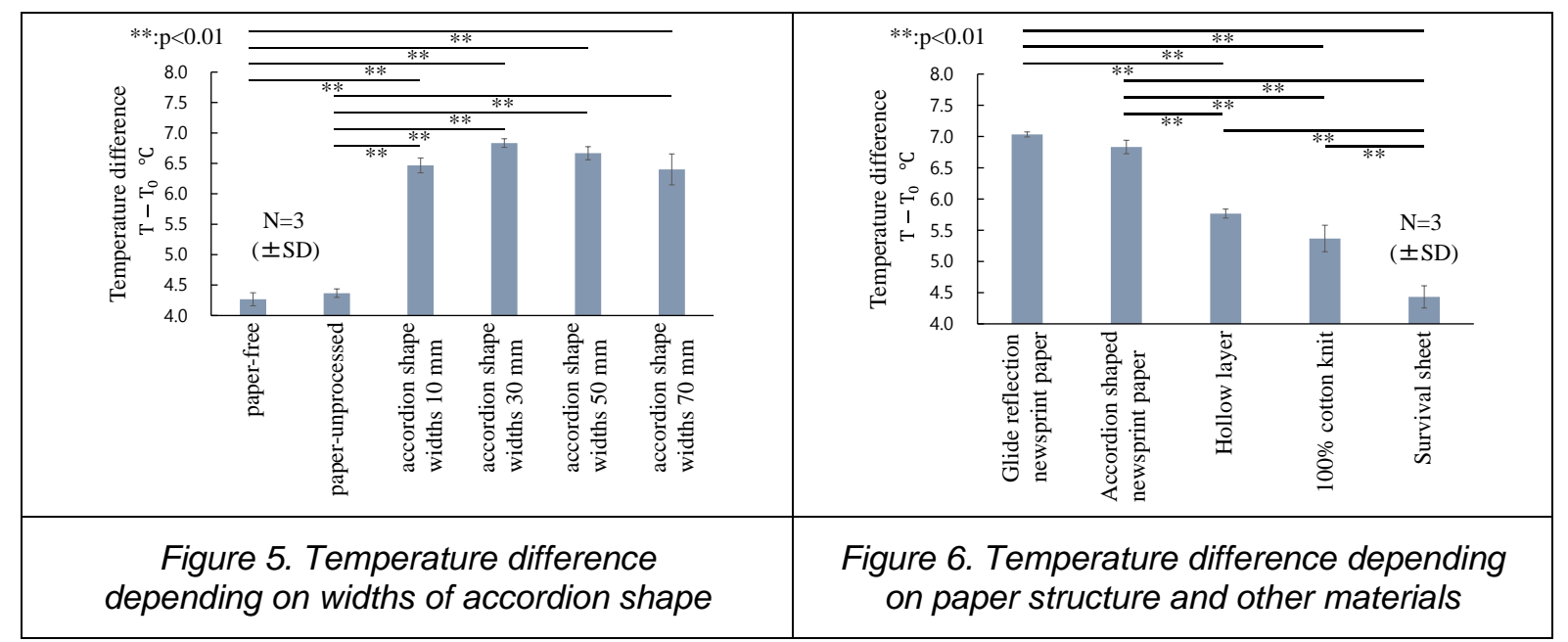

\subsubsection{Experiment and results on the apex angle of folded face to face}

We investigated the effect of the apex angle of the folded face to face in accordion shape and glide reflection structure on the heat retention based on the method described in 3.1.2. The experimental conditions are described below. The angle of the accordion shape was $\theta$ as shown in Figure 4, and was set to every $20^{\circ}$ in the range of $20-120^{\circ}$, with six conditions. The angle of the glide reflection structure was $\theta$ as shown in Figure 2, and was set in the same way as for the accordion shape for six conditions. The width of the accordion shape was set as $15 \mathrm{~mm}, a$ shown in Figure 4, under the same conditions as in the previous experiments. The width of the glide reflection was set as $54 \mathrm{~mm}, b=27$ mm shown in Figure 2, based on the results of the structural analyses described in Chapter 4 below. Three test pieces were tested for each condition.

Figure 7 shows the experimental results and the error bar indicates standard deviation. The effect of the angle on the temperature difference in the accordion shapes and glide reflection structures is small, except for $\theta=120^{\circ}$. The reason for this is thought to be the effect of the volume of air being held. Comparing the volume of the air layer in the accordion shape at $\theta=20^{\circ}$ and $\theta=120^{\circ}$, where a significant difference occurs, the volume at $\theta=120^{\circ}$ is $49 \%$ of that at $\theta=20^{\circ}$. In other words, when the increase of the angle reduces the height of the structure, the volume of air contained reduces for suppressing heat transfer. Meanwhile, in a glide reflection structure, it is thought that these effects are unlikely to occur, because each structure holds the sufficient volume of air comparing to in accordion shape with $\theta=120^{\circ}$. Even the smallest air volume in a glide reflection structure is 1.7 times as large as that in an accordion shape at $\theta=120^{\circ}$.

\subsection{Design of dimensions based on rigidity under pressure of clothes}

\subsubsection{Model of a glide reflection structure}

To verify whether this glide reflection structure has sufficient rigidity to maintain the air layer under the pressure of the clothes, we investigated the dimensions of the structure with the highest rigidity within the range of expected heat retention effects. To create a glide reflection structure, a single facing sheet of 
newsprint paper $(\mathrm{V}, \mathrm{H})$ was cut in two, and one of the sheets $(\mathrm{V}, \mathrm{H} / 2)$ was folded in half lengthwise $(\mathrm{V}, \mathrm{H} / 4)$. A three-dimensional model of the structure was constructed for each combination of the fold width $b \mathrm{~mm}$, and the angle between the folded surface $\theta^{\circ}$, and a computer simulation of structural analyses was considered. The length of the fold width $b \mathrm{~mm}$ is determined by the following constraints.

- Constraint 1. It must be within the range of air layer sizes expected to have a high heat retention effect as indicated in 3.1.

- Constraint 2. Few newsprint papers should be wasted during creating the glide reflection structure.

First, we narrowed down the conditions for the width $b \mathrm{~mm}$ from constraint 1 . The results of 3.1.3 indicated that the widths of an accordion shape with a relatively high heat retention effect were $30 \mathrm{~mm}$ and $50 \mathrm{~mm}$. The height of the air layer of the structure with width $a=30 \mathrm{~mm}$ is $26 \mathrm{~mm}$, and the height of that with width $a=50 \mathrm{~mm}$ is $45 \mathrm{~mm}$. Therefore, the length of the fold width $b \mathrm{~mm}$ was set within this range to produce the height of the air layer is between 26 and $45 \mathrm{~mm}$.

Then, from constraint 2, we narrowed down the conditions for the length of the width $b \mathrm{~mm}$. In order to prevent paper chips from wasting in constructing a glide reflection structure, the length of the long side of a newsprint paper, approx. $540 \mathrm{~mm}$, should be divisible by the width of the glide reflection $b$ $\mathrm{mm}$. Therefore, the candidate widths $b$ of the glide reflection structure are 27.0, 28.4, 30.0, 31.8, 33.8, $36.0,38.6,41.5$ and $45.0 \mathrm{~mm}$. At $27.0 \mathrm{~mm}(2 b=54 \mathrm{~mm})$, it is possible to make 10 units of glide reflection structure on newsprint paper, and at $45.0 \mathrm{~mm}$, it is possible to make 6 units.

The apex angle $\theta^{\circ}$ of the folded face to face was set to every $20^{\circ}$ in the range of 40 to $100^{\circ}$, which was indicated to have no significant difference in the heat retention effect based on the results in 3.1.5. Table 1 shows the material properties of the newsprint paper used in the analyses.

The 3D model described above is applied a load corresponding to the pressure of clothes during use. The load of $0.4678 \mathrm{~N} / \mathrm{mm}^{2}$, based on melton used for coat, was applied to the top edge of the structure in the negative z-axis direction as shown in Figure 8. The convex portion of the glide reflection structure is fixed and the fold is constrained as hinge.

These conditions are applied to 36 3D-models and the maximum displacement in the z-axis direction $[\mathrm{mm}]$ is calculated. The rigidity of the structure under each condition was calculated from the external force $[\mathrm{N}]$ divided by the maximum displacement $[\mathrm{mm}]$, and the rigidity is thought to be a value in the normal direction to the body when the paper structure covers the trunk of the body.

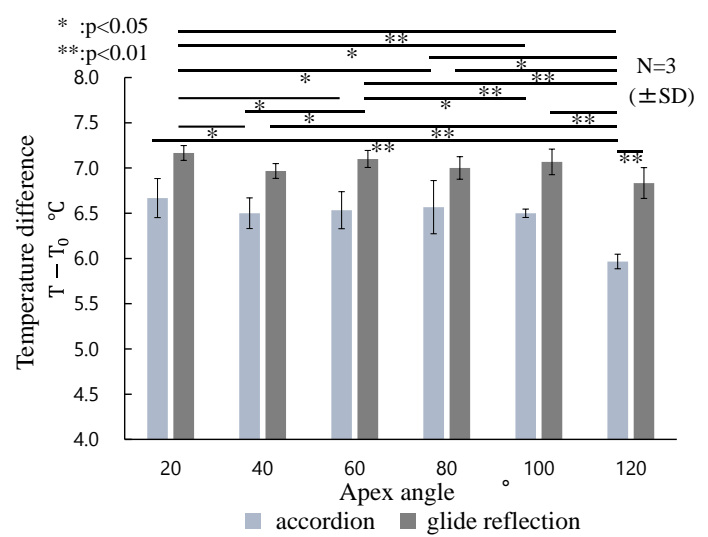

Table 1. Dimensions and material properties of newsprint paper

\begin{tabular}{|c|c|}
\hline Property: A3, half hold & Value \\
\hline Thickness & $0.07 \times 2=0.14$ \\
\hline Density & 0.623 \\
\hline Young's modulus (MD) GPa & 5.2 \\
\hline Young's modulus (CD) GPa & 1.1 \\
\hline Poisson's ratio & 0.38 \\
\hline
\end{tabular}

MD: machine direction. $\mathrm{CD}$ : cross direction

Figure 7. Temperature difference depending

on the apex angle of folded face to face

\subsubsection{Structural analyses of glide reflection structures}

For all combinations of fold widths and face-to-face angles, the maximum displacement in the z-axis occurred in the centre of the top edge of the structure. Figure 9 shows the calculated results of the rigidity of the structure. The results of these analyses show that the rigidity of the structure is high at fold width $27 \mathrm{~mm}$ for all conditions of $\theta^{\circ}$. In the previous experiments (Hodozuka, 2018), it has been confirmed that approx. $0.13 \mathrm{~N} / \mathrm{mm}$ at most is sufficient to maintain the folded-paper structure when it is inserted between clothes.

\section{PERFORMANCE EXPERIMENT}

\subsection{Heat retention experiment comparing uses for newsprint paper}

From the results of Chapter 3, the length of the fold width, $b=27 \mathrm{~mm}$, of the glide reflection structure 


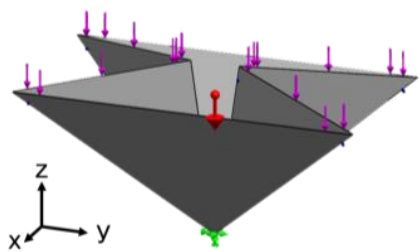

Figure 8. Loading and restraint conditions in structural analysis

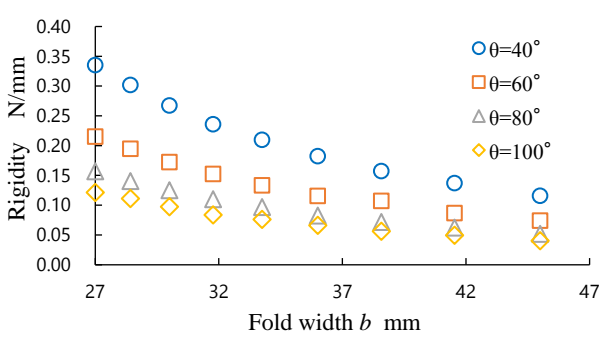

Figure 9. Results of structural analyses depending on fold widths and apex angles

was determined. We created a full-scale temporary cold protective gear by using this structure and investigated the heat retention effects by using a thermal manikin to compare to the conditions without newsprint paper and previous structure. The experiment was conducted based on the method for measuring the thermal resistance of clothing with a thermal manikin, as specified in ISO 9920. Thermal resistance $R{ }^{\circ} \mathrm{C} \cdot \mathrm{m}^{2} / W$ is calculated from dividing the difference between surface temperature of thermal manikin $t_{s}{ }^{\circ} \mathrm{C}$, located in a constant temperature and humidity room and wearing a cold protective gear, and room temperature $t_{a}{ }^{\circ} \mathrm{C}$ by heat supplied to the thermal manikin $H_{d} \mathrm{~W} / \mathrm{m}^{2}$.

$$
R=\left(t_{s}-t_{a}\right) / H_{d}
$$

As shown in Figure 10 and 11, a cold protective gear of newsprint paper was inserted between a t-shirt as underwear and a shirt as outerwear around the abdomen of thermal manikin to simulate practical use. A thermocouple (2) for measuring the surface temperature of thermal manikin was attached at the position shown in Figure 10. A thermocouple (1) was placed at a distance of $1500 \mathrm{~mm}$ horizontally to the floor from the thermocouple (2) to measure the room temperature. The four conditions were as follows: (i) only the underwear and outerwear were worn without newsprint paper, (ii) unprocessed newsprint paper was additionally worn, (iii) newsprint paper with folded-plate structure $(15 \mathrm{~mm}$ width of accordion shape) was additionally worn, and (iv) newsprint paper with glide reflection structure was additionally worn, as shown in Figure 11. In order to cover the same area around the abdomen of thermal manikin, the number of newsprint paper used was (ii) 2 spreads on unprocessed newsprint paper, (iii) 3 spreads on folded-plate structure, and (iv) 4.5 spreads on glide reflection structure. The room temperature is set at $15^{\circ} \mathrm{C}$ and the thermal manikin is under comfort control (a control method like that of the human body in which the surface temperature varies depending on the surrounding environment). The temperature was determined at the time when the temperature doesn't fluctuate for $30 \mathrm{~min}$ and reaches equilibrium after wearing each test piece. The thermal resistance $R_{0}{ }^{\circ} \mathrm{C} \cdot \mathrm{m}^{2} / W$ without any clothes and $R{ }^{\circ} \mathrm{C} \cdot \mathrm{m}^{2} / W$ with clothes and each newsprint paper structure are calculated based on the formula (1) and compared among the conditions. The same test pieces were used in each condition, and were measured three times per condition.

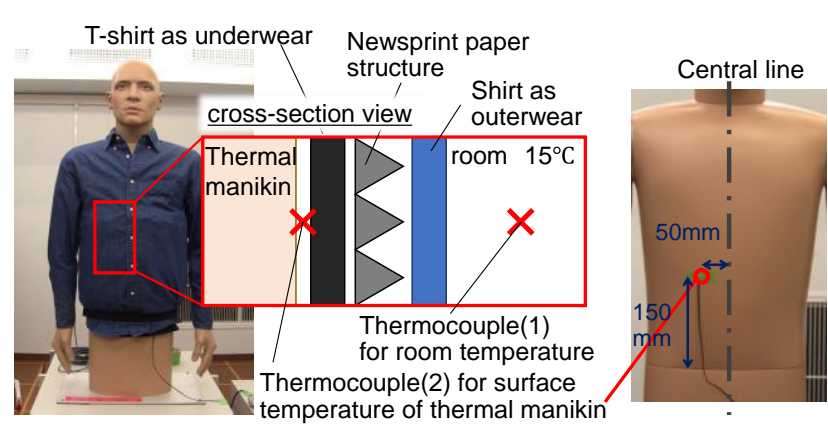

Figure 10. Environment of the heat retention effect experiment

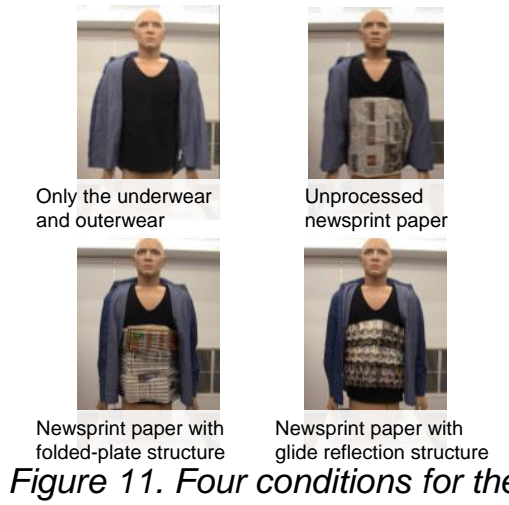

Figure 11. Four conditions for the heat retention effect experiment

\subsection{Results of heat retention experiment comparing uses for newsprint paper}

Figure 12 shows that the thermal resistance was significantly higher in the unprocessed newsprint paper condition than in the clothes-only condition, and in the paper structure conditions than in the unprocessed paper condition. Furthermore, the thermal resistance of glide reflection structure was significantly higher than that of folded-plate structure.

A folded-plate structure, as shown in Figure 1, includes many straight lines along the surface of the body, which may allow air to flow in and out of the gaps. Another possible effect is that the basic unit 
is smaller than the glide reflection structural unit in order to maintain the air layer under the pressure of the clothes, therefore, the folded-plate structure holds less air than the glide reflection structure.

Finally, we investigated whether the structure covers around aluminum plates with radii of $40,80,120$, 160,200 , and $220 \mathrm{~mm}$ corresponding to that of human body. It was confirmed that the mountain tops of the structure were in contact with the surface of the aluminum plate at all radii. This indicates the structure can be applied to various body shapes.

\section{CONSIDERATIONS}

By folding newsprint paper into a glide reflection structure, the heat retention effect is improved and the rigidity under the pressure of the clothes is increased. On the other hand, compared to the folded-plate structure, it is extremely difficult to create a glide reflection structure without making preparations such as drawing fold lines on newsprint paper. When the author first created the glide reflection structure, it took $2 \mathrm{~h}$ to draw the fold lines on newsprint paper with a ruler and pen, and moreover $2 \mathrm{~h}$ to actually fold along the lines, for a total of $4 \mathrm{~h}$. In order to use this idea in practice, it is necessary to simplify the creation process. Therefore, we consulted the process for making the Miura fold and have developed an assistive tool that enables newsprint paper to be easily folded into a glide reflection structure for a temporary cold protective gear. This tool consists of a flexible silicone sheet and a magnetic sheet traced to face of a glide reflection structure, placed in the glide reflection folds. This tool sandwiches newsprint paper inside and makes a user to fold the paper easily into a glide reflection structure. Using this tool, the author was able to create a temporary cold protective gear unit in about 30 to $60 \mathrm{~min}$. Figure 13 shows how newsprint paper is actually folded into a glide reflection structure through using an assistive tool, and a temporary cold protective gear unit made by using the tool.

Finally, we discuss scenarios in which the idea of paper structure can be used on site. First is a way of spreading the idea before actual evacuation life. The use of newsprint paper for heat retention has already been introduced on the websites of various local governments as wisdom for living in shelters. The scenario is to add how to fold on those websites for making it widely available. Then, the printed pattern paper will be included in disaster prevention booklets distributed by local governments, or the pattern paper will be printed on newspaper for National Disaster Prevention Day and stored in each household.

The same applies to an assistive tool for folding a glide reflection structure. The material and the magnetic shape of the tool will be also posted on the websites so that people can make their own tools. In addition, a workshop will be organised on how to make the folding assistive tool and the cold protective gear as part of disaster prevention exercise in school and in municipality.

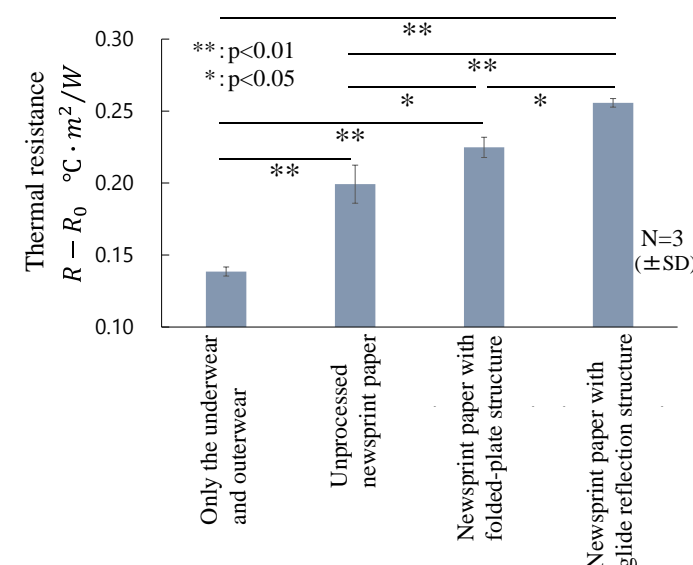

Figure 12. Thermal resistance depending on newsprint paper structures

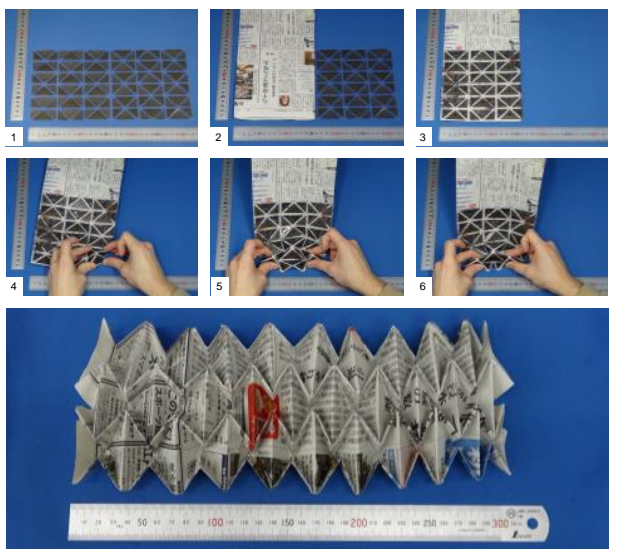

Figure 13. Assistive tool for folding glide reflection structure and a temporary cold protective gear unit

The last is a way of sharing the idea directly with evacuees during evacuation life. As an example, there is a website that collects and publishes variety of ideas on wisdom of living including how to secure drinking water, how to make lanterns, etc. at the time of the Great East Japan Earthquake. (OLIVE, 2011). For those who could not browse the website, volunteer activities were carried out to print out booklets of information, transport them to the sites, and distribute them. This approach will be also used to share the idea to create a cold protective gear on site.

The idea of paper folding structure can also be applied to other short commodities such as underwear and clothes, and will broaden the applicable scope in relation to resource-sensitive design, and the 
benefits will be directly provided to society. Then, the investigation of the effective range of heat retention and rigidity, and the optimized structure will be studied further, and the design-ofexperiments approach and the optimization design will be applied at the time. This will also contribute further to advances in origami engineering.

\section{CONCLUSION}

Necessary supplies are often short during the evacuation life after a natural disaster. Especially during the cold season, the cold protective products tend to be insufficient in evacuation centres. If people can make and wear simple cold protective gear by using materials obtainable on site, it will reduce the burden on the evacuees, even if only temporarily. In order to address these problems, the authors' group has been working on the development of cold protective gear that can be made at evacuation sites.

We focused on newsprint paper, which is already being used to improve the heat retention effect as an easily available material and as empirical use. The basic principle of the heat retention effect is to create a layer of air with low thermal conductivity. In order to maintain the air layer under the pressure of the clothes with as few pieces as possible, the idea of folding the newsprint paper into an accordion shape was devised previously. In this study, we investigated an alternative structure of newsprint paper, which can improve the heat retention effect and be applied to various body shapes. Therefore, we focused on the glide reflection structure repeating a smaller chamber. The basic size was determined by experiments with reference to the accordion shape, and the experimental results indicated that the heat retention effect was significantly greater than that of a mere air layer and those of ordinary fabrics. Furthermore, it was found that the apex angle of folded face to face had no significant difference in the heat retention effect. Finally, the dimensions of the structure were determined to maintain the air layer under the pressure of the clothes by simulation of structural analyses.

Furthermore, we made a temporary cold protective gear that can practically cover the body trunk and investigated the heat retention effect by using a thermal manikin. It was confirmed that the thermal resistance was significantly higher than that of unprocessed newsprint paper and that of accordion shape, i.e., the heat retention effect was enhanced. Finally, an assistive tool to create temporary cold protective gear with glide reflection structure and scenarios that the idea can be used on site were also considered.

\section{REFERENCES}

Cabinet Office, Government of Japan. (2013), "Disaster Management Measures: Let's start with what we can do!", DISASTER MANAGEMENT NEWS, No.73, p.18. http://www.bousai.go.jp/kohou/kouhoubousai/h25/ 73/pdf/bs13winter.pdf (accessed 2021-03-22)

Cabinet Office, Government of Japan. (2013), The report on survey results of promotion of comprehensive measures for evacuation, p.44-49. http://www.bousai.go.jp/kaigirep/houkokusho/hinan_taisaku/pdf/ hinan_taisaku_houkokusyo.pdf (accessed 2021-03-22)

Committee of Infrastructure Planning and Management. (2016), Kumamoto Earthquake Survey Report, https:// jsce-ip.org/wp-content/uploads/2019/03/06d1263347a8ec6b0ec9e3d5f50956dd.pdf (accessed 2021-03-22)

Edholm, O.,G. (1978), Man: Hot and Cold (Studies in Biology), Hodder

Hodozuka, H. and Wesugi, S. (2016), "Improving Heat-Retaining Property of Newsprint Paper Utilized for Life at Evacuation Centers Following Disasters", Human Interface Symposium 2016, p.213-216.

Hodozuka, H., Oda, T. and Wesugi, S. (2018), "Designing a Temporary Cold Protective Gear with HeatRetaining Property and Rigidity Using Newsprint Paper”, Design \& Systems Conference 2018, 2603, https://doi.org/10.1299/jsmedsd.2018.28.2603

OLIVE. (2011), https://nosigner.com/olive (accessed 2021-03-22)

Reconstruction Agency. (2012), The report on disaster-related deaths in the Great East Japan Earthquake, https://www.reconstruction.go.jp/topics/20120821_shinsaikanrenshihoukoku.pdf (accessed 2021-03-22)

Saito, H. (1974), Architecture and climate, Kyoritsu shuppan

Smith, C.E. (2007), Design For The Other 90\%, Cooper Hewitt, Smithsonian Design Museum

Tokyo Metropolitan Government. (2015), Disaster preparedness tokyo, https://www.bousai.metro.tokyo.lg.jp/1002147/1008042/1008046/index.html (accessed 2021-03-22)

Turner, N., Goodwine, B. and Sen, M. (2016), "A review of origami applications in mechanical engineering", Proc IMechE Part C: J Mechanical Engineering Science, 2016, Vol. 230(14), pp.2345-2362. 